\title{
Association of Baseline Neutrophil-to-Lymphocyte Ratio with Clinicopathological Characteristics of Papillary Thyroid Carcinoma
}

\author{
Dimitrios K. Manatakis, ${ }^{1}$ Sofia Tseleni-Balafouta, ${ }^{2}$ Dimitrios Balalis, ${ }^{1}$ Vasiliki N. Soulou, ${ }^{1}$ \\ Dimitrios P. Korkolis, ${ }^{1}$ George H. Sakorafas, ${ }^{1}$ Georgios Plataniotis, ${ }^{1}$ and \\ Emmanouil Gontikakis ${ }^{1}$
}

${ }^{1}$ First Department of Surgical Oncology, St. Savvas Cancer Hospital, Athens, Greece

${ }^{2}$ Department of Pathology, School of Medicine, National and Kapodistrian University of Athens, Athens, Greece

Correspondence should be addressed to Dimitrios K. Manatakis; dmanatak@yahoo.gr

Received 8 February 2017; Revised 17 March 2017; Accepted 21 March 2017; Published 9 May 2017

Academic Editor: Jack Wall

Copyright (c) 2017 Dimitrios K. Manatakis et al. This is an open access article distributed under the Creative Commons Attribution License, which permits unrestricted use, distribution, and reproduction in any medium, provided the original work is properly cited.

\begin{abstract}
Objective. To investigate the potential association of neutrophil-to-lymphocyte ratio (NLR), a surrogate systemic inflammatory biomarker, with clinical and pathological characteristics of papillary thyroid cancers. Methods. 205 patients with papillary carcinoma were identified from the institutional thyroid cancer database between 2006 and 2015 (55 males, 150 females, mean age $51.2 \pm 14.7$ years). NLR was calculated as the absolute neutrophil count divided by the absolute lymphocyte count, based on the preoperative complete blood cell counts. Results. NLR was significantly higher in carcinomas with extrathyroidal invasion $(2.74 \pm 01.24$ versus $2.39 \pm 0.96, p=0.04)$ and bilateral $(2.67 \pm 1.15$ versus $2.35 \pm 0.96, p=0.03)$ and multifocal tumours $(2.65 \pm 1.08$ versus $2.29 \pm 0.96, p=0.01)$, as well as lymph node-positive tumours $(3.12 \pm 1.07$ versus $2.41 \pm 1.02, p=0.03)$. On the other hand, NLR values were not associated with gender, age, tumour size, histologic subtype, the presence of thyroiditis, and TNM staging. Conclusions. As an index of inflammation, NLR is inexpensive, readily available, and easy to extract from routine blood tests. We found increased NLR values in papillary carcinomas with poorer histopathological profile and more aggressive clinical behaviour. Whether this systemic inflammatory response, as expressed by the NLR, represents the inflammatory microenvironment leading to tumourigenesis, or is a tumour-associated phenomenon, remains to be elucidated and warrants further study.
\end{abstract}

\section{Introduction}

Tumourigenesis is the result of dysregulation of a delicate balance between elaborate cancer-promoting and cancerinhibiting molecular pathways. Inflammation plays a critical role in all aspects of cancer, including development, progression, and metastasis, and this complex interplay between neoplastic cells and their inflammatory microenvironment may have significant influence on patient prognosis [1]. Inflammatory cells have probably a protean dual role, pro- and anticarcinogenic, depending on the circumstances of tumour/host interactions. This missing link between inflammation and tumour biology and course remains to be elucidated.

Papillary thyroid carcinoma (PTC), the most common endocrine malignancy, exhibits a close association with chronic inflammation; however, recent studies have produced inconsistent results concerning inflammation and tumour behaviour. An increased incidence of papillary carcinomas is observed in patients with thyroiditis, although chronic lymphocytic thyroiditis may ultimately correlate with improved prognosis in patients with PTC [2].

Several studies to date have shown that inflammatory biomarkers (CRP, TNFa, IL-6, neutrophil-to-lymphocyte 
ratio) reliably predict poor prognosis in a variety of malignant neoplasms (oesophageal, gastric, pancreatic, colonic, ovarian, renal, and lung) [1-3]. As a surrogate inflammatory marker, neutrophil-to-lymphocyte ratio (NLR) is readily available and easy to extract from routine blood tests. However, its use in the preoperative assessment and postoperative follow-up of PTC cases is still limited and controversial.

Initial studies reported higher NLR in larger tumours and older patients, and generally higher NLR predicted higher risk of recurrence and poor prognosis [3-5]. Other investigators however failed to reproduce these results $[6,7]$. Moreover, wide variations in proposed cutoff values contribute to this ongoing debate $[5,8]$.

The aim of our study was to evaluate a possible association of preoperative NLR with clinicopathological characteristics of patients with PTC, in order to determine its status as a potential biomarker in the pretreatment setting.

\section{Materials and Methods}

Patients diagnosed with PTC between January 2006 and December 2015 at the First Department of Surgical Oncology, St. Savvas Cancer Hospital, were retrospectively identified from the institutional thyroid surgery database. Demographic characteristics (age, sex, and comorbidity), laboratory test results, and pathology outcomes were documented for each patient.

Included in the analysis were all consecutive patients, aged $\geq 18$ years, who underwent thyroidectomy for papillary carcinoma $(>1.1 \mathrm{~cm})$ or microcarcinoma $(\leq 1.0 \mathrm{~cm})$, either suspected by the preoperative FNA biopsy or confirmed in the final histopathology. Excluded were patients with known haematological disorders, chronic medical conditions affecting white blood cell counts, past history of malignancy, acute myocardial infarction or coronary revascularisation within 6 months before surgery, and glucocorticoid administration within 3 months before surgery. Noneuthyroid patients, patients with acute infections or with baseline total WBC count outside the institutional reference range $(4000-10,000 / \mathrm{ml})$, were also excluded.

Neutrophil-to-lymphocyte ratio was calculated as the absolute neutrophil count divided by the absolute lymphocyte count, based on the preoperative complete blood cell count. According to our preoperative assessment protocol, fasting baseline blood samples are routinely obtained between 08:00 and 10:00 am on the day before surgery and include haematocrit, haemoglobin, total WBC and automated differential counts (neutrophils, lymphocytes, monocytes, basophils, and eosinophils) and platelets. This standardised protocol contributed to adjusting for the known impact of circulating hormones (circadian rhythm) on the number and distribution of WBC subtypes.

Pathology reports were reviewed independently by two authors to determine thyroid specimen weight, tumour size, uni- or multifocality, uni- or bilaterality, lymph node metastasis, extrathyroidal invasion, TNM stage according to AJCC 7th edition, presence of thyroiditis, and PTC histologic subtype (classic versus follicular variant). Other variants (diffuse sclerosing, tall cell, columnar, oncocytic, solid, clear
TABLE 1: Demographic characteristics and haematological data of PTC patients.

\begin{tabular}{lcc}
\hline$N$ & 205 & \\
Sex & & \\
$\quad$ Male & 55 & $26.8 \%$ \\
$\quad$ Female & 150 & $73.2 \%$ \\
Age (years) & $51.2 \pm 14.7$ & Range $18-89$ \\
$\quad<45$ years & 65 & $31.7 \%$ \\
$\quad \geq 45$ years & 140 & $68.3 \%$ \\
Thyroid specimen weight (gr) & $32.7 \pm 37.46$ & Range 5-329 \\
TSH (mIU/L) & $1.45 \pm 1.48$ & Range $0.29-4.19$ \\
WBC total (cells/mL) & $7527 \pm 2166$ & Range $4200-10,000$ \\
Neutrophils (cells/mL) & $4744 \pm 1767$ & Range $1900-9100$ \\
Lymphocytes (cells/mL) & $2084 \pm 661$ & Range $900-5200$ \\
NLR (mean \pm SD) & $2.44 \pm 1.02$ & Range $0.86-6.14$ \\
\hline
\end{tabular}

cell, cribriform morular, hobnail, and dedifferentiated) were much more rarely encountered and therefore precluded any meaningful comparisons. Patient subgroups based on clinicopathological parameters were compared in terms of total WBC, neutrophil, and lymphocyte counts as well as NLR values. Tumour size was determined according to the greatest diameter of the lesion based on the postoperative histopathology report. Multifocal tumours were defined as 2 or more foci of PTC in the surgical specimen. Bilateral tumours were defined as PTC lesions affecting both thyroid lobes, in contrast to unilateral tumours, in which lesions were found unilaterally. Finally, thyroiditis was identified by lymphocytic infiltration and formation of lymph follicles and included both cases of Hashimoto's and nonspecific lymphocytic thyroiditis.

On the basis of NLR stratification, patients were subsequently divided into two subgroups by the median NLR value, the lymphophilic low NLR and the neutrophilic high NLR groups. These groups were compared in terms of the aforementioned clinicopathological characteristics.

Continuous variables were expressed as mean \pm standard deviation, while categorical variables were expressed as frequencies or percentages. Statistical analysis was performed on SPSS, version 20.0, using Student's $t$-test and ANOVA for continuous variables and chi-square test for categorical variables. Statistical significance was set to $p<0.05$. The study was approved by the institutional research ethics committee.

\section{Results}

During the course of the study period, 233 patients were diagnosed with PTC. Of those, 205 patients fulfilled the inclusion criteria and were included in the final analysis. Demographic characteristics and haematological data are shown in Table 1. Association of the preoperative total WBC, neutrophils, lymphocytes, and NLR with the clinicopathological characteristics of PTC is depicted in Table 2. 
TABLE 2: Association of preoperative total WBC, neutrophils, lymphocytes, and NLR with clinicopathological characteristics of PTC.

\begin{tabular}{|c|c|c|c|c|c|c|c|c|c|}
\hline & $N$ & Total WBC & $p$ value & Neutro & $p$ value & Lympho & $p$ value & NLR & $p$ value \\
\hline \multicolumn{10}{|l|}{ Sex } \\
\hline Male & 55 & $7682 \pm 2131$ & & $4868 \pm 1787$ & & $2034 \pm 723$ & & $2.62 \pm 1.19$ & \\
\hline Female & 150 & $7495 \pm 2179$ & 0.31 & $4714 \pm 1765$ & 0.31 & $2111 \pm 637$ & 0.26 & $2.38 \pm 0.96$ & 0.09 \\
\hline \multicolumn{10}{|l|}{ Age } \\
\hline$<45$ years & 65 & $7857 \pm 2637$ & & $5070 \pm 2268$ & & $2053 \pm 602$ & & $2.58 \pm 1.07$ & \\
\hline$\geq 45$ years & 140 & $7419 \pm 1934$ & 0.12 & $4628 \pm 1510$ & 0.07 & $2105 \pm 684$ & 0.33 & $2.39 \pm 1.01$ & 0.15 \\
\hline \multicolumn{10}{|l|}{ Tumour size } \\
\hline $0.1-1 \mathrm{~cm}$ & 113 & $7421 \pm 2161$ & & $4686 \pm 1850$ & & $2051 \pm 558$ & & $2.40 \pm 0.96$ & \\
\hline $1.1-2 \mathrm{~cm}$ & 57 & $7682 \pm 2332$ & & $4741 \pm 1639$ & & $2277 \pm 879$ & & $2.32 \pm 1.10$ & \\
\hline $2.1-4 \mathrm{~cm}$ & 29 & $7880 \pm 2105$ & & $5020 \pm 1822$ & & $2060 \pm 588$ & & $2.61 \pm 1.16$ & \\
\hline$>4.1 \mathrm{~cm}$ & 6 & $7600 \pm 935$ & 0.81 & $5200 \pm 752$ & 0.82 & $1540 \pm 518$ & 0.07 & $3.55 \pm 0.73$ & 0.12 \\
\hline \multicolumn{10}{|l|}{ Tumour size } \\
\hline $0.1-1 \mathrm{~cm}$ & 113 & $7421 \pm 2161$ & & $4686 \pm 1850$ & & $2051 \pm 558$ & & $2.40 \pm 0.96$ & \\
\hline$>1.1 \mathrm{~cm}$ & 92 & $7738 \pm 2164$ & 0.18 & $4864 \pm 1638$ & 0.27 & $2152 \pm 793$ & 0.17 & $2.51 \pm 1.13$ & 0.26 \\
\hline \multicolumn{10}{|c|}{ Extrathyroidal invasion } \\
\hline Yes & 43 & $7438 \pm 1987$ & & $4779 \pm 1753$ & & $1897 \pm 569$ & & $2.74 \pm 1.24$ & \\
\hline No & 162 & $7548 \pm 2208$ & 0.4 & $4744 \pm 1774$ & 0.46 & $2118 \pm 675$ & 0.05 & $2.39 \pm 0.96$ & 0.04 \\
\hline \multicolumn{10}{|l|}{ Multifocality } \\
\hline Yes & 91 & $7684 \pm 2259$ & & $4983 \pm 1920$ & & $1999 \pm 623$ & & $2.65 \pm 1.08$ & \\
\hline No & 114 & $7444 \pm 2093$ & 0.24 & $4589 \pm 1636$ & 0.08 & $2157 \pm 681$ & 0.06 & $2.29 \pm 0.96$ & 0.01 \\
\hline \multicolumn{10}{|l|}{ Bilaterality } \\
\hline Yes & 65 & $7860 \pm 2337$ & & $5127 \pm 2039$ & & $2042 \pm 621$ & & $2.67 \pm 1.15$ & \\
\hline No & 140 & $7413 \pm 2080$ & $<0.01$ & $4601 \pm 1625$ & 0.04 & $2110 \pm 677$ & 0.27 & $2.35 \pm 0.96$ & 0.03 \\
\hline \multicolumn{10}{|c|}{ Lymph node metastasis } \\
\hline Yes & 15 & $7050 \pm 1089$ & & $4800 \pm 1235$ & & $1613 \pm 331$ & & $3.12 \pm 1.07$ & \\
\hline No & 190 & $7570 \pm 2201$ & 0.25 & $4754 \pm 1793$ & 0.47 & $2115 \pm 664$ & 0.02 & $2.41 \pm 1.02$ & 0.03 \\
\hline \multicolumn{10}{|l|}{ Histologic subtype } \\
\hline Classic & 70 & $7450 \pm 1986$ & & $4658 \pm 1597$ & & $2069 \pm 608$ & & $2.41 \pm 0.97$ & \\
\hline Follicular variant & 135 & $7607 \pm 2275$ & 0.33 & $4819 \pm 1874$ & 0.29 & $2104 \pm 694$ & 0.37 & $2.47 \pm 1.07$ & 0.36 \\
\hline \multicolumn{10}{|l|}{ Thyroiditis } \\
\hline No & 88 & $7297 \pm 2093$ & & $4538 \pm 1662$ & & $2049 \pm 608$ & & $2.36 \pm 0.98$ & \\
\hline Nonspecific & 90 & $7976 \pm 2167$ & & $5113 \pm 1892$ & & $2173 \pm 686$ & & $2.54 \pm 1.10$ & \\
\hline Hashimoto & 27 & $7226 \pm 2279$ & 0.14 & $4530 \pm 1681$ & 0.13 & $2008 \pm 760$ & 0.45 & $2.48 \pm 1.03$ & 0.59 \\
\hline \multicolumn{10}{|l|}{ T stage (AJCC) } \\
\hline T1a & 107 & $7407 \pm 2168$ & & $4666 \pm 1849$ & & $2055 \pm 559$ & & $2.38 \pm 0.95$ & \\
\hline $\mathrm{T} 1 \mathrm{~b}$ & 37 & $8027 \pm 2545$ & & $4977 \pm 1731$ & & $2381 \pm 965$ & & $2.32 \pm 1.02$ & \\
\hline $\mathrm{T} 2$ & 17 & $7780 \pm 1708$ & & $4890 \pm 1194$ & & $2150 \pm 700$ & & $2.44 \pm 0.86$ & \\
\hline $\mathrm{T} 3$ & 44 & $7500 \pm 1829$ & 0.61 & $4814 \pm 1731$ & 0.86 & $1928 \pm 582$ & 0.07 & $2.76 \pm 1.30$ & 0.34 \\
\hline \multicolumn{10}{|l|}{ TNM stage (AJCC) } \\
\hline I & 169 & $7588 \pm 2268$ & & $4474 \pm 1861$ & & $2121 \pm 664$ & & $2.40 \pm 1.00$ & \\
\hline II & 10 & $7920 \pm 944$ & & $5440 \pm 573$ & & $1760 \pm 297$ & & $3.14 \pm 0.48$ & \\
\hline III & 21 & $7073 \pm 1642$ & & $4340 \pm 1134$ & & $1967 \pm 704$ & & $2.50 \pm 1.20$ & \\
\hline IVa & 5 & $6675 \pm 645$ & 0.67 & $4600 \pm 868$ & 0.66 & $1400 \pm 163$ & 0.09 & $3.37 \pm 1.05$ & 0.11 \\
\hline
\end{tabular}

The bold data represent statistically significant $p$ values.

NLR values did not differ in conjunction with gender or age ( $<45$ or $\geq 45$ years). Although we observed a tendency towards higher NLR in larger tumours (especially $>4.1 \mathrm{~cm}$ ), this difference did not reach statistical significance. NLR was also not significantly different, when microcarcinomas were compared to macro-carcinomas. On the other hand, multifocal and bilateral PTC showed higher $\operatorname{NLR}(2.65 \pm 1.08$ versus $2.29 \pm 0.96, p=0.01$ and $2.67 \pm 1.15$ versus $2.35 \pm 0.96, p=0.03$, resp.), as did tumours with extrathyroidal invasion $(2.74 \pm 01.24$ versus $2.39 \pm 0.96$, 
TABLE 3: Comparison of prevalence of prognostic factors in the low and high NLR subgroups.

\begin{tabular}{lccc}
\hline & $\begin{array}{c}\text { Low NLR } \\
(<2.17)\end{array}$ & $\begin{array}{c}\text { High NLR } \\
(>2.17)\end{array}$ & $p$ value \\
\hline$N$ & 102 & 103 & \\
Sex & & & \\
$\quad$ Male & $25(24.5 \%)$ & $30(29.1 \%)$ & \\
$\quad$ Female & $77(75.5 \%)$ & $73(70.9 \%)$ & 0.46 \\
Age (years) & $52.74 \pm 13.18$ & $51.15 \pm 15.45$ & 0.24 \\
Specimen weight (gr) & $26.01 \pm 18.24$ & $38.52 \pm 43.39$ & $\mathbf{0 . 0 0 9}$ \\
Tumour size (cm) & $0.95 \pm 0.69$ & $1.30 \pm 1.43$ & 0.38 \\
Extrathyroidal & $20(19.6 \%)$ & $23(22.3 \%)$ & 0.63 \\
invasion & & & \\
Lymph node & $3(2.9 \%)$ & $12(11.7 \%)$ & $\mathbf{0 . 0 3}$ \\
metastasis & & & \\
Multifocality & $43(42.1 \%)$ & $48(46.6 \%)$ & 0.52 \\
Bilaterality & $30(29.4 \%)$ & $35(33.9 \%)$ & 0.48 \\
Thyroiditis & $52(50.9 \%)$ & $65(63.1 \%)$ & 0.08 \\
Follicular variant & $68(66.7 \%)$ & $67(65 \%)$ & 0.81 \\
T stage & & & \\
$\quad$ T1a + T1b & $75(73.5 \%)$ & $69(67 \%)$ & \\
$\quad$ T2 + T3 & $27(26.5 \%)$ & $34(33 \%)$ & 0.31 \\
\hline
\end{tabular}

The bold data represent statistically significant $p$ values.

$p=0.04)$ and positive lymph nodes (3.12 \pm 1.07 versus $2.41 \pm 1.02, p=0.03)$. Finally, baseline NLR was not associated with PTC subtype, thyroiditis, or TNM stage.

Comparison of the low versus high NLR subgroups (Table 3) showed again that age and sex did not correlate with NLR; however, high NLR values were associated with larger goitres (38.52 versus $26.01 \mathrm{gr}, p=0.009$ ). Tumour size, extrathyroidal invasion, multifocality, bilaterality, presence of thyroiditis, PTC variant, and T stage also did not differ between the groups. On the contrary, cases with lymph node metastases were more frequently encountered in the high than in the low NLR group ( $11.7 \%$ versus $2.9 \%, p=0.03)$.

\section{Discussion}

The potential association between chronic inflammation and cancer was first suggested by German pathologist Rudolf Virchow more than a century ago [1]. The underlying mechanisms have been extensively investigated during the past decade, and gastrointestinal malignancies are a fine example of this connection (chronic H. pylori gastritis and gastric cancer, chronic viral hepatitis $\mathrm{B}$ and $\mathrm{C}$ and hepatocellular carcinoma, idiopathic inflammatory bowel disease and colorectal cancer) [9].

In the field of thyroid research, the relationship between chronic lymphocytic thyroiditis and PTC was first proposed by Dailey in 1955 [10]. Indeed, pathologists have long recognised that certain tumours are densely infiltrated by a mixture of macrophages and lymphocytes (cells of the innate and adaptive immune systems, resp.) both within and around the primary tumour [11].
The innate immune system comprises of mechanisms that protect the host in a nonspecific manner and is mediated by natural killer cells, macrophages, dendritic cells, mast cells, and polymorphonuclear leukocytes (neutrophils, basophils, and eosinophils) [11]. Neutrophils are important mediators of the inflammatory response, and blood neutrophilia has long been recognised as a marker of systemic inflammatory response [1]. On the other hand, lymphocytes of the adaptive immune system have evolved to provide a more versatile defense system plus increased protection following reinfection with the same pathogen [11]. Specifically in cases of malignancy, tumour-associated neutrophilia and/or lymphopenia is considered either a paraneoplastic manifestation or a nonspecific response to cancer-related inflammation due to local tissue destruction and cytokine release [11].

At the molecular level, upregulation of the RET/RAS/ BRAF/ERK/MAPK pathway, which has the capability to induce both a proinflammatory and a protumourigenic thyroid programme, is a possible mechanism linking chronic thyroiditis with carcinogenesis [9]. Recent research has further suggested that papillary carcinomas harbour a different genetic background according to the association or not with thyroiditis (expression of BRAFV600E in cases without and expression of RET/PTC in cases with autoimmunity) [12]. This is also supported by evidence showing that the RET/ PTC oncogene can activate a transcriptional proinflammatory programme in normal thyrocytes [13].

This tumour-host interaction, in the form of systemic inflammatory response, is generally not taken into account in most contemporary prognostic systems [3]. However, inflammatory biomarkers (CRP, cytokines, and white cell counts) can be independent prognostic factors in a variety of tumours (oesophageal, gastric, pancreatic, colorectal, mesothelioma, ovarian, renal, and bladder) [2].

Neutrophil-to-lymphocyte ratio has recently emerged as a simple and valid composite marker of systemic inflammatory response [3]. Although it is inexpensive, easily calculated, and readily available, its use in the preoperative assessment and postoperative follow-up of PTC patients remains a matter of debate. To date, studies examining NLR in well-differentiated thyroid cancer were heterogeneous in statistical methodology, varied largely in their sample sizes (41-3364 PTC cases), and produced inconsistent results (Table 4 ).

In general, higher NLR values were variably associated with larger tumour size $[3,5,6,14]$, multifocality [14], lymph node metastases [14], and higher TNM stage [4, 6, 14], indicating a more aggressive tumour behaviour and more advanced disease stage. Our study confirmed this relation of high NLR with poor tumour profile, in terms of extrathyroidal invasion, multifocality, bilaterality, and lymph node metastasis.

On the contrary, advanced age ( $\geq 45$ years) has been previously correlated both with lower [15] and with higher $[4,6]$ NLR values. In our series, PTC cases $\geq 45$ years had a slightly lower NLR $(2.39 \pm 1.01)$ compared to $<45$ years $(2.58 \pm 1.07)$, the difference however was not significant $(p=0.15)$.

Interestingly, the only study demonstrating a statistically significant correlation between NLR and the presence of 
TABLE 4: Studies examining NLR in association with clinicopathological characteristics of PTC.

\begin{tabular}{|c|c|c|}
\hline & Association found & No association found \\
\hline Age & $\begin{array}{l}\text { Liu et al. [4] (lower NLR in patients }<45 \text { years) } \\
\text { Lang et al. [6] (lower NLR in patients }<45 \text { years) } \\
\text { Kim et al. [15] (lower NLR in patients } \geq 45 \text { years) }\end{array}$ & $\begin{array}{c}\text { Liu et al. [3] } \\
\text { Kim et al. [5] } \\
\text { Gong et al. [14] } \\
\text { Manatakis et al. }\end{array}$ \\
\hline Sex & & $\begin{array}{c}\text { Liu et al. [3], Liu et al. [4], Kim et al. [5], } \\
\text { Lang et al. [6], Gong et al. [14] } \\
\text { Manatakis et al. }\end{array}$ \\
\hline Tumour size & $\begin{array}{l}\text { Liu et al. [3], Kim et al. [5], } \\
\text { Lang et al. [6], Gong et al. [14] }\end{array}$ & $\begin{array}{c}\text { Liu et al. [4], Cho et al. [8], } \\
\text { Kim et al. [15], Manatakis et al. }\end{array}$ \\
\hline Extrathyroidal invasion & Manatakis et al. & $\begin{array}{c}\text { Liu et al. [3], Liu [4] Kim et al. [5], } \\
\text { Lang et al. [6], Kim et al. [15] }\end{array}$ \\
\hline Multifocality & Gong et al. [14], Manatakis et al. & $\begin{array}{l}\text { Liu et al. [3], Kim et al. [5], Lang et al. [6], } \\
\text { Kim et al. [15] }\end{array}$ \\
\hline Bilaterality & Manatakis et al. & Lang et al. [6] \\
\hline Lymphovascular invasion & & Liu et al. [3], Lang et al. [6], Kim et al. [15] \\
\hline Lymph node metastasis & Gong et al. [14], Manatakis et al. & $\begin{array}{l}\text { Liu et al. [3], Liu et al. [4], Kim et al. [5], } \\
\text { Lang et al. [6], Kim et al. [15] }\end{array}$ \\
\hline TNM stage & $\begin{array}{l}\text { Liu et al. [4] (stages I-II versus III-IV) } \\
\text { Lang et al. [6] (for stage I) } \\
\text { Gong et al. [14] (for patients } \geq 45 \text { years) }\end{array}$ & $\begin{array}{c}\text { Liu et al. [3] } \\
\text { Kim et al. [5] } \\
\text { Kim et al. [15] } \\
\text { Manatakis et al. }\end{array}$ \\
\hline Histologic subtype & & Lang et al. [6], Manatakis et al. \\
\hline Thyroiditis & Kim et al. [15] (lower NLR in Hashimoto) & $\begin{array}{c}\text { Liu et al. [3], Lang et al. [6], } \\
\text { Kocer et al. [16], Manatakis et al. }\end{array}$ \\
\hline Recurrence risk & Liu et al. [3] & \\
\hline Prognosis & Kim et al. [5] (for stages III-IV) & Lang et al. [6], Cho et al. [8] \\
\hline
\end{tabular}

chronic Hashimoto thyroiditis found only a subtle difference in NLR values (1.52 with versus 1.58 without Hashimoto), obviously of minimal clinical importance [15]. Liu et al. and Kocer et al. both showed a trend towards lower NLR values in PTC cases with concurrent chronic lymphocytic thyroiditis $[3,16]$. Indeed, several studies have reported an increased incidence of well-differentiated thyroid cancer in patients with thyroiditis, although these patients paradoxically seem to have a better prognosis [17, 18]. Our series could not establish an association between NLR and Hashimoto thyroiditis (NLR $2.48 \pm 1.03$ with versus $2.36 \pm 0.96$ without thyroiditis, $p=0.59)$.

An interesting point, confirmed also in this study, is the relatively low NLR values of papillary thyroid cancer, in comparison to other solid tumours. A comprehensive metaanalysis, investigating the association of NLR with patient prognosis in a variety of neoplasms (gastrointestinal, gynaecological, urological, and pulmonary; head and neck, brain, and breast), found indeed NLR medians up to 7.7 [19]. Ratios above the cutoff correlated with unfavourable outcome and worse prognosis, in terms of overall survival (median NLR cutoff 4, range 1.9-7.2), cancer-specific survival (median NLR cutoff 3.85, range 1.9-5.0), progression-free survival (median NLR cutoff 3, range 2.0-5.0), and disease-free survival (median NLR cutoff 5.0, range 2.0-7.7). Our cohort exhibited a mean NLR of $2.44 \pm 1.02$ and a median NLR of 2.17 (range 0.86-6.14). Furthermore, only 12 patients $(5.9 \%)$ presented with ratios greater than 4 . Similar results have been obtained in all studies dealing with NLR in welldifferentiated thyroid cancer (Table 5). Even in anaplastic carcinomas, which represent the most aggressive form of thyroid neoplasia, a diagnostic cutoff of 3.8 is proposed, to aid discrimination against well-differentiated cancers [8].

This phenomenon has been attributed to the fact that inflammation may be less important in the initiation of thyroid carcinogenesis and to the relatively indolent nature of differentiated thyroid cancer, which causes a less vigorous systemic inflammatory response [3]. In any case, NLR remains largely a nonspecific biomarker of systemic inflammation. Although promising in terms of sensitivity, careful exclusion of all patients with medical conditions affecting white blood cell differential counts (acute infections, allergic reactions, cardiovascular incidents etc.) is mandatory. Nevertheless, the NLR is universally available from routine blood tests and does not increase the preoperative diagnostic work-up cost [2].

Limitations of our study protocol include its singleinstitution, retrospective nature and average sample size. Furthermore, our study could not incorporate the most recent changes in terminology of the follicular variant of PTC [20]. The subgroup "follicular variant" was inevitably heterogeneous and included both cases of infiltrative and encapsulated follicular variant PTC. Modern molecular profiling however has indicated that the latter closely resembles follicular adenoma/follicular carcinoma, whereas the former behaves like the classic PTC. Indeed noninvasive, 
TABLE 5: Mean or median NLR values in studies on PTC (N/R not reported).

\begin{tabular}{|c|c|c|c|c|}
\hline Name & Year & Number of PTC cases & NLR (mean or median) & Range \\
\hline Liu et al. [3] & 2012 & 159 & 1.94 & $1.50-2.57$ \\
\hline Seretis et al. [2] & 2013 & 52 & $\begin{array}{l}3 \text { in PTMC } \\
3.4 \text { in PTC }\end{array}$ & $\mathrm{N} / \mathrm{R}$ \\
\hline Kim et al. [5] & 2013 & 542 & 1.74 & $\mathrm{~N} / \mathrm{R}$ \\
\hline Lang et al. [6] & 2014 & 191 & 2.68 & $0.52-16.6$ \\
\hline Liu et al. [4] & 2015 & 321 & $\begin{array}{l}1.99 \text { in }<45 \text { years } \\
2.28 \text { in }>45 \text { years }\end{array}$ & $0.4-8.58$ \\
\hline Cho et al. [8] & 2015 & 3364 & 1.86 & $0.28-16.29$ \\
\hline Kocer et al. [16] & 2015 & 65 & $\begin{array}{l}2.47 \text { with thyroiditis } \\
2.57 \text { without thyroiditis }\end{array}$ & $\mathrm{N} / \mathrm{R}$ \\
\hline Kim et al. [15] & 2015 & 1066 & $\begin{array}{l}1.75 \text { in }<45 \text { years } \\
1.52 \text { in } \geq 45 \text { years }\end{array}$ & $0.25-10.20$ \\
\hline Gong et al. [14] & 2016 & 161 & 2 & $0.64-6.38$ \\
\hline Yaylaci et al. [7] & 2016 & 41 & 1.9 & $\mathrm{~N} / \mathrm{R}$ \\
\hline Manatakis et al. & & 205 & 2.44 & $0.86-6.14$ \\
\hline
\end{tabular}

encapsulated follicular variant of PTC has been reclassified as "Noninvasive follicular thyroid neoplasm with papillarylike nuclear features" [20]. We therefore consider that such retrospective comparison between classic and follicular variants of PTC may probably be subject to bias and inaccuracy.

Finally, our study protocol was neither designed to address the issue of NLR in conjunction with patient prognosis nor to propose specific cutoff values. Given the generally excellent 5-year and 10-year disease-free and overall survival of PTC patients, a long-term follow-up would be required to draw safe, meaningful conclusions. Only 3 studies have reported long-term patient outcomes, with a mean followup of 60 months $[5,6,8]$. While Kim et al. showed worse disease-free survival in stage III-IV patients with an NLR cutoff of 1.5, Lang et al. and Cho et al. found that NLR determined neither disease-free survival nor cancer-specific death rates $[5,6,8]$. On the other hand, the association of higher NLR with aggressive clinical and pathological characteristics may imply unfavourable outcome. Our aim is to closely monitor these patients over the following years, in order to assess the prognostic value of NLR in PTC cases.

Implications for future study include also investigating the possible association between intra- and peritumoural immune cell infiltration with systemic white blood cell counts, as well as the expected normalisation of postthyroidectomy levels of the NLR.

\section{Conclusion}

Whether increased NLR in more aggressive tumours represents the inflammatory microenvironment leading to tumourigenesis, or is a tumour-associated phenomenon, remains to be elucidated. Whereas NLR was significantly increased in invasive, bilateral, multifocal, and lymph nodepositive papillary cancers, our study found no difference in NLR values between patients with and without chronic lymphocytic thyroiditis. As a surrogate systemic inflammatory biomarker, NLR is inexpensive, readily available, and warrants further study in larger trials.

\section{Conflicts of Interest}

The authors declare that there is no conflict of interests regarding the publication of this paper.

\section{References}

[1] M. M. Moore, W. Chua, K. A. Charles, and S. J. Clarke, "Inflammation and cancer: causes and consequences," Clinical Pharmacology and Therapeutics, vol. 87, no. 4, pp. 504-508, 2010.

[2] C. Seretis, S. Gourgiotis, G. Gemenetzis, F. Seretis, E. Lagoudianakis, and G. Dimitrakopoulos, "The significance of neutrophil/lymphocyte ratio as a possible marker of underlying papillary microcarcinomas in thyroidal goiters: a pilot study," American Journal of Surgery, vol. 205, no. 6, pp. 691696, 2013.

[3] C.-L. Liu, J. Lee, T. Liu, Y. Chang, Y.-C. Hsu, and S.-P. Cheng, "Blood neutrophil-to-lymphocyte ratio correlates with tumor size in patients with differentiated thyroid cancer," Journal of Surgical Oncology, vol. 107, no. 5, pp. 493-497, 2013.

[4] J. Liu, J. Du, J. Fan et al., "The neutrophil-to-lymphocyte ratio correlates with age in patients with papillary thyroid carcinoma," Orl, vol. 77, no. 2, pp. 109-116, 2015.

[5] J.-Y. Kim, T. Park, S.-H. Jeong et al., "Prognostic importance of baseline neutrophil to lymphocyte ratio in patients with advanced papillary thyroid carcinomas," Endocrine, vol. 46, no. 3, pp. 526-531, 2014.

[6] B. H. H. Lang, C. P. C. Ng, K. B. Au, K. P. Wong, K. K. C. Wong, and K. Y. Wan, "Does preoperative neutrophil lymphocyte ratio predict risk of recurrence and occult central nodal metastasis in papillary thyroid carcinoma?" World Journal of Surgery, vol. 38, no. 10, pp. 2605-2612, 2014.

[7] S. Yaylaci, O. Tosun, O. Sahin et al., "Lack of variation in inflammatory hematological parameters between benign 
nodular goiter and papillary thyroid cancer," Asian Pacific Journal of Cancer Prevention, vol. 17, no. 4, pp. 2321-2323, 2016.

[8] J. Cho, M. Park, Y. Ryu, and J. Yoon, "The neutrophil to lymphocyte ratio can discriminate anaplastic thyroid cancer against poorly or well differentiated cancer," Annals of Surgical Treatment and Research, vol. 88, no. 4, pp. 187-192, 2015.

[9] A. Bozec, S. Lassalle, V. Hofman, M. Ilie, J. Santini, and P. Hofman, "The thyroid gland: a crossroad in inflammationinduced carcinoma? An ongoing debate with new therapeutic potential," Current Medicinal Chemistry, vol. 17, no. 30, pp. 3449-3461, 2010.

[10] L. S. Ward, "Immune response in thyroid cancer: widening the boundaries," Scientifica (Cairo), vol. 2014, Article ID 125450, 20 pages, 2014.

[11] L. L. Cunha, M. A. Marcello, and L. S. Ward, "The role of the inflammatory microenvironment in thyroid carcinogenesis," Endocrine-Related Cancer, vol. 21, no. 3, pp. R85-R103, 2014.

[12] M. Muzza, D. Degl'Innocenti, C. Colombo et al., “The tight relationship between papillary thyroid cancer, autoimmunity and inflammation: clinical and molecular studies," Clinical Endocrinology (Oxford), vol. 72, no. 5, pp. 702-708, 2010.

[13] M. G. Borrello, L. Alberti, A. Fischer et al., "Induction of a proinflammatory program in normal human thyrocytes by the RET/PTC1 oncogene," Proceedings of the National Academy of Sciences of the United States of America, vol. 102, no. 41, pp. 14825-14830, 2005.

[14] W. Gong, S. Yang, X. Yang, and F. Guo, "Blood preoperative neutrophil-to-lymphocyte ratio is correlated with TNM stage in patients with papillary thyroid cancer," Clinics (São Paulo, Brazil), vol. 71, no. 6, pp. 311-314, 2016.

[15] S. M. Kim, E. H. Kim, B. H. Kim et al., "Association of the preoperative neutrophil-to-lymphocyte count ratio and platelet-to-lymphocyte count ratio with clinicopathological characteristics in patients with papillary thyroid cancer," Endocrinology and Metabolism, vol. 30, no. 4, pp. 494-501, 2015.

[16] D. Kocer, C. Karakukcu, H. Karaman, F. Gokay, and F. Bayram, "May the neutrophil/lymphocyte ratio be a predictor in the differentiation of different thyroid disorders?" Asian Pacific Journal of Cancer Prevention, vol. 16, no. 9, pp. 38753879, 2015.

[17] S. D. Larson, L. N. Jackson, T. S. Riall et al., "Increased incidence of well-differentiated thyroid cancer associated with Hashimoto Thyroiditis and the role of the PI3k/Akt pathway," Journal of the American College of Surgeons, vol. 204, no. 5, pp. 764-773, 2007.

[18] E. Kebebew, P. A. Treseler, P. H. G. Ituarte, and O. H. Clark, "Coexisting chronic lymphocytic thyroiditis and papillary thyroid cancer revisited," World Journal of Surgery, vol. 25, no. 5, pp. 632-637, 2001.

[19] A. J. Templeton, M. G. McNamara, B. Šeruga et al., "Prognostic role of neutrophil-to-lymphocyte ratio in solid tumors: a systematic review and meta-analysis," Journal of the National Cancer Institute, vol. 106, no. 6, pp. 1-11, 2014.

[20] G. Tallini, R. M. Tuttle, and R. A. Ghossein, "The history of the follicular variant of papillary thyroid carcinoma," Journal of Clinical Endocrinology and Metabolism, vol. 102, no. 1, pp. 15-22, 2017. 


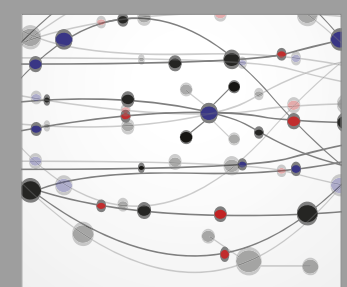

The Scientific World Journal
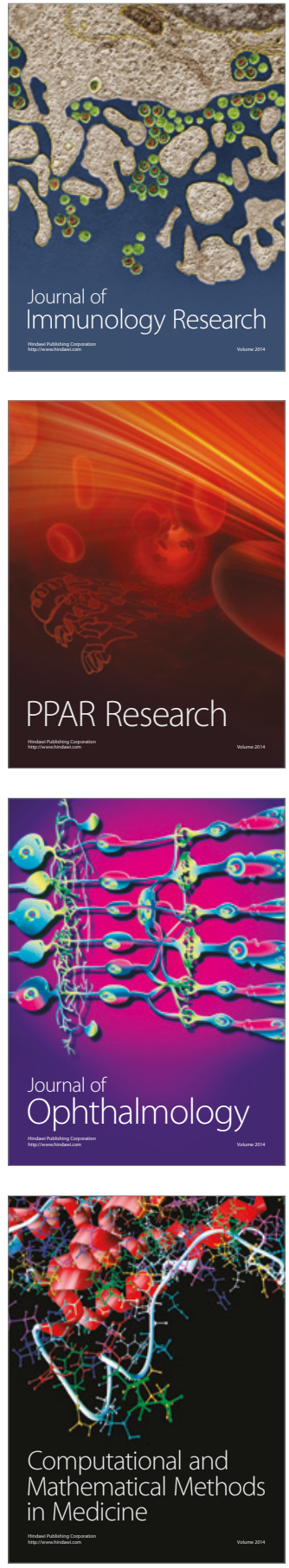

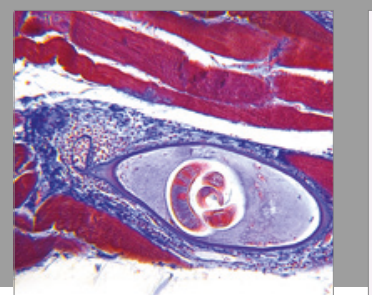

Gastroenterology Research and Practice
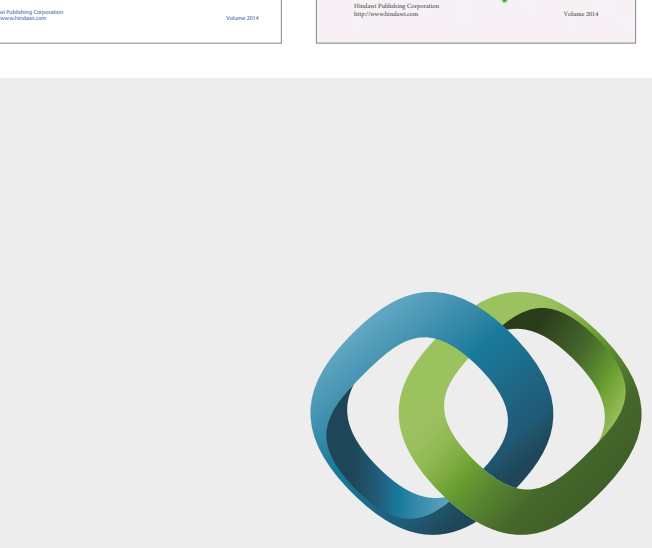

\section{Hindawi}

Submit your manuscripts at

https://www.hindawi.com
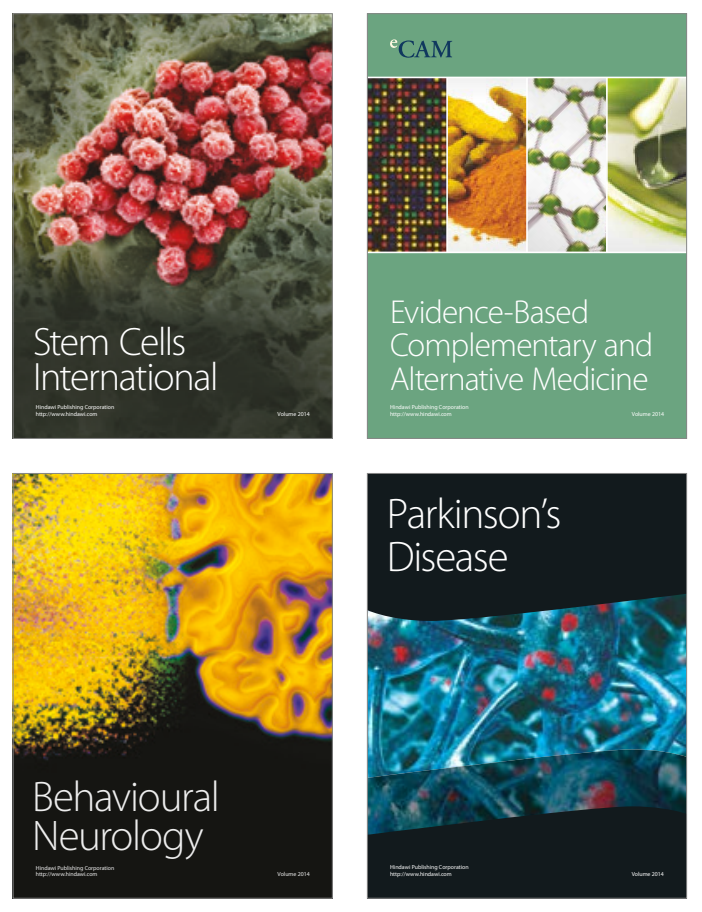
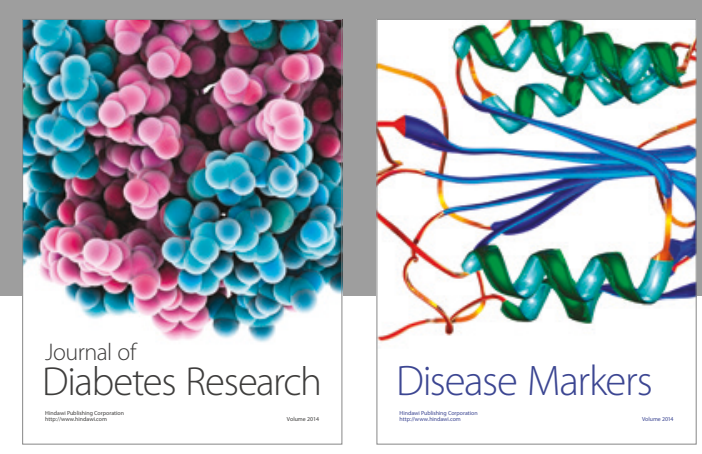

Disease Markers
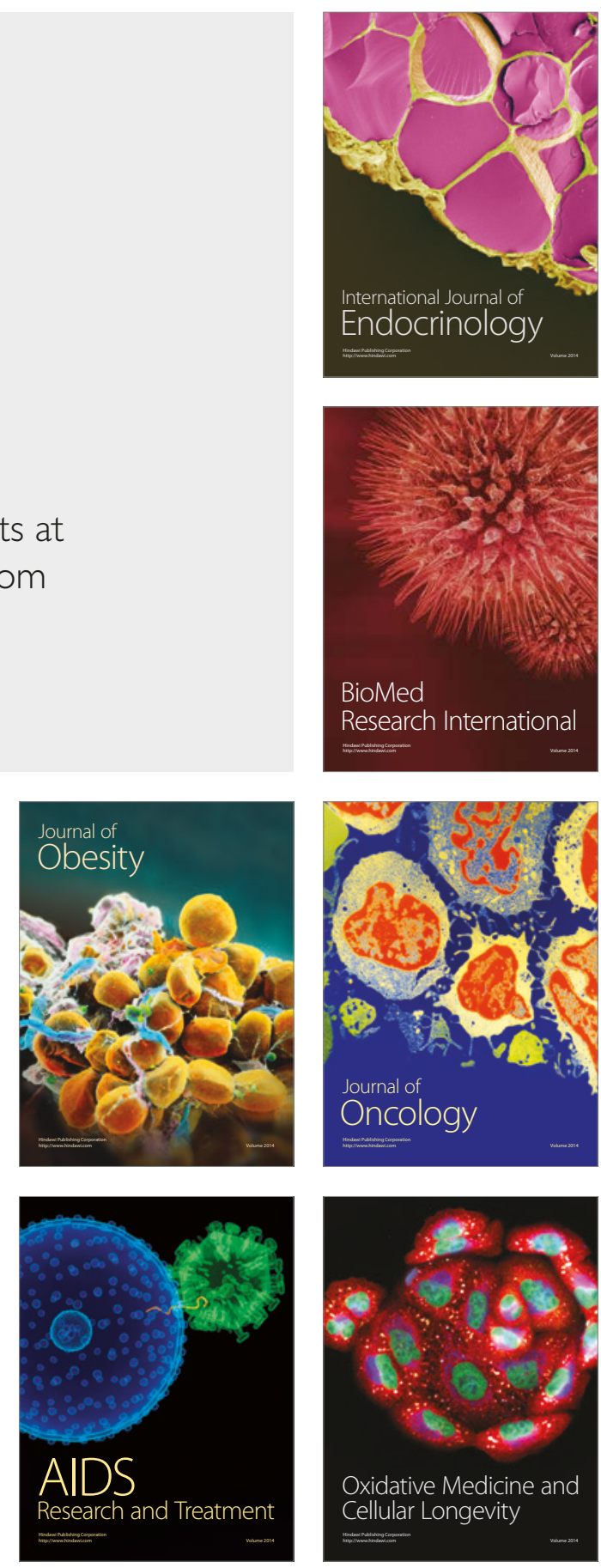